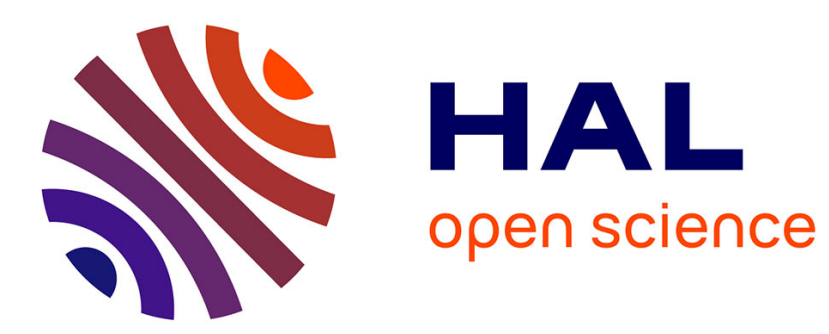

\title{
State estimation for polytopic LPV descriptor systems: application to fault diagnosis
}

\author{
Habib Hamdi, Mickael Rodrigues, Chokri Mechmeche, Didier Theilliol, \\ Naceur Benhadj Braiek
}

\section{- To cite this version:}

Habib Hamdi, Mickael Rodrigues, Chokri Mechmeche, Didier Theilliol, Naceur Benhadj Braiek. State estimation for polytopic LPV descriptor systems: application to fault diagnosis. 7th IFAC Symposium on Fault Detection, Supervision and Safety of Technical Processes, Safeprocess 2009, Jun 2009, Barcelone, Spain. 10.3182/20090630-4-ES-2003.00073 . hal-00422479

HAL Id: hal-00422479

https://hal.science/hal-00422479

Submitted on 7 Oct 2009

HAL is a multi-disciplinary open access archive for the deposit and dissemination of scientific research documents, whether they are published or not. The documents may come from teaching and research institutions in France or abroad, or from public or private research centers.
L'archive ouverte pluridisciplinaire HAL, est destinée au dépôt et à la diffusion de documents scientifiques de niveau recherche, publiés ou non, émanant des établissements d'enseignement et de recherche français ou étrangers, des laboratoires publics ou privés. 


\title{
State Estimation for Polytopic LPV Descriptor Systems: Application to Fault Diagnosis
}

\author{
H.HAMDI ${ }^{*}$ M. RODRIGUES ${ }^{* *}$ C. MECHMECHE ${ }^{*}$ \\ D. THEILLIOL ${ }^{* * *}$ N. BENHADJ BRAIEK* \\ * Laboratoire d'Etude et Commande Automatique des Processus \\ (LECAP), Ecole Polytechnique de Tunisie; (e-mail: \\ chokri.mechmeche@esstt.rnu.tn,naceur.benhadj@ept.rnu.tn). \\ ** Laboratoire d'Automatique et de Génie des Procédés (LAGEP), \\ CNRS UMR 5007 Université Lyon 1, France (e-mail: \\ rodrigues@lagep.cpe.fr) \\ *** Centre de Recherche en Automatique de Nancy (CRAN), CNRS \\ UMR 7039 Université Nancy 1, France, (e-mail: \\ didier.theilliol@cran.uhp-nancy.fr)
}

\begin{abstract}
In this paper, the design of a polytopic Unknown Input Observer (UIO) for polytopic Linear Varying Parameter (LPV) descriptor systems is investigated. The method presented is based on the representation of affine LPV descriptor systems where parameters evolve in a hypercube domain. The considered polytopic UIO is able to estimate the states of the system in spite of the presence of unknown inputs. This approach is also applied to actuator fault detection, isolation and estimation. Stability conditions of such observer are expressed in terms of Linear Matrix Inequalities (LMI). An example illustrates the effectiveness and performances of such polytopic LPV observer.
\end{abstract}

Keywords: Nonlinear descriptor systems, multi-model, unknown inputs observer, fault diagnosis, linear matrix inequalities $\left(\mathcal{L} \mathcal{M} \mathcal{I}_{\mathrm{s}}\right)$.

\section{INTRODUCTION}

Real physical processes are often described by nonlinear models. As it is difficult to design nonlinear observers, many authors preferred to represent these systems by a Linear Parameter Varying (LPV) approach. The idea of this approach is to represent the system as an interpolation of simple (usually affine) local models.

For affine LPV systems, the interpolation techniques present a good approach to get a polytopic structure. This structure is a set of linear model scheduled by a convex function. Several attempts have already been made in this direction. Taking the polytopic representation, in (Rodrigues et al.2008), (Akhenak et al 2004) and (Rodrigues et al.2005), the authors have developed a method for fault diagnosis and have designed a robust polytopic unknown input observer. In the same area, (Sylvain et al.2006), (Zolghadri et al.2008) have applied this approach in the design of Fault Detection and Isolation filters for varying parameters models.

Many processes are naturally modeled by systems of nonlinear Differential and Algebraic Equations (DAE), where the explicit differential equations arise from dynamic balances of mass and energy, while the algebraic equations typically consist of empirical correlations. DAE, singular or descriptor systems arise frequently in various engineering fields as well, especially in chemical, mechanical and electrical engineering.
Observer design for such descriptor nonlinear systems has been studied by many authors as (Boutayeb et al.1995) who have proposed an asymptotic observer of autonomous nonlinear descriptor systems where the matrix $\mathrm{E}$ is in the general form and may be rectangular, (Darouach et al.1995), (Hou et al.1999) for their works on the design of observer for descriptor systems . A full-order and proportional integral observers for lipschitz nonlinear descriptor systems have been designed by (Koenig et al.2006).

For nonlinear descriptor time invariant systems, (Marx et al 2007) have proposed a multiobserver by extending the ordinary Takagi-Sugeno (T-S) fuzzy model. Finally, nonlinear descriptor systems are often studied for control (Taniguchi et al.2000), robust control (Wang et al.2004), (Toscano 2006) and fault tolerant control purposes (Gao et al.2007). Now, we will focus on systems which can be represented by polytopic LPV models (Rodrigues et al.2007). We will use this representation and extend it to descriptor polytopic LPV systems.

In this paper, we study the design of unknown inputs observers (UIO) for polytopic LPV descriptor systems. This observer is used for state estimation and for residual generation in order to detect and isolate the faults when they occur (see (Chen et al.1999) for Fault Diagnosis preliminaries).

The first step of this work consists to represent the affine LPV descriptor system by a polytopic form when the parameters evolve in a polytopic domain. The vertices of this 
polytope are called the submodels of this representation. Those submodels are then combined by weighing functions to yield a global model. The second step is devoted to the design of a local observer for each singular submodel. Stability of this polytopic UIO is ensured by using LMI techniques.

This paper is organized as follows. The polytopic representation of affine LPV singular systems is presented in Section 2. The polytopic Unknown Input Observer design corresponding to the polytopic models is given in Section 3 . The stability conditions of the polytopic UIO are studied in Section 4. Section 5 is dedicated to detect, isolate and estimate an actuator fault by using a bank of UIO. Finally, an illustrative example is provided to show the effectiveness of the proposed approach.

\section{POLYTOPIC LPV STRUCTURAL DESCRIPTOR SYSTEM}

Let us consider the following LPV descriptor representation

$$
\left\{\begin{array}{l}
E \dot{x}(t)=A(\theta) x(t)+B(\theta) u(t)+R(\theta) d(t) \\
y(t)=C x(t)
\end{array}\right.
$$

where $x \in R^{n}$ is the state vector, $u \in R^{p}$ the input vector, $d \in R^{q}$ is the unknown inputs vector and $y \in R^{m}$ represents the measured outputs vector.

$\left(\mathrm{E}, \mathrm{A}_{\mathrm{i}}\right) \in R^{\mathrm{lxn}}, \mathrm{E}$ is a constant matrix and $\operatorname{rank}(\mathrm{E})<\mathrm{n}$. $\Theta=\left\{\theta_{1}, \ldots, \theta_{r}\right\}$ is the vector of $\mathrm{r}$ parameters. In the case of parameters affine dependence, the elements of the representation (1) can be written as follows

$$
\Pi(\theta)=\Pi_{0}+\sum_{i=1}^{r} \theta_{i} \Pi_{i}
$$

where

$$
\Pi(\theta)=\left(\begin{array}{ccc}
A(\theta) & B(\theta) & R(\theta) \\
C & 0 & 0
\end{array}\right), \forall \theta \in \Theta
$$

where $\Theta=\left\{\theta: \theta=\theta_{1}, \ldots, \theta_{r} \in \Theta \subset R^{r}\right\}$.

In this paper, the field $\Theta$ is an hypercube of dimension $r$, where each vertex corresponds to the extreme values of $\theta$ (Rodrigues et al 2007). The system (1) can be defined via barycentric combination of a matrix polytope with vertices $S_{i}=\left[A_{i}, B_{i}, C, R_{i}\right]$,

$\forall i \in[1, \ldots, M]$ where $M=2^{r}$. Consequently, system (1) can be rewritten as a polytopic representation

$$
\left\{\begin{array}{l}
E \dot{x}(t)=\sum_{i=1}^{M} \mu_{i}(\theta(t))\left(A_{i} x(t)+B_{i} u(t)+R_{i} d(t)\right) \\
y(t)=C x(t)
\end{array}\right.
$$

where $\mu_{i}(\theta(t))=\mu\left(\bar{\theta}_{i}, \underline{\theta}_{i}, \theta_{i}(t), t\right)\left(\bar{\theta}_{i}\right.$ and $\underline{\theta}_{i}$ represent the maximum and the minimum value of $\theta_{i}$ respectively). $A_{i} \in R^{n \times n}, B_{i} \in R^{n \times p}, R_{i} \in R^{n \times q}$ and $C \in R^{m \times n}$ are time invariant matrices defined for the $i^{\text {th }}$ model. The polytopic LPV descriptor system is scheduled through $\mu_{i}(\theta(t)), \forall i \in[1, \ldots, M]$ functions lie in the following convex set

$$
\Omega=\left\{\begin{array}{c}
\mu_{i}(\theta(t)) \in R^{M}, \mu_{i}(\theta)=\left[\mu_{1}(\theta), \ldots, \mu_{M}(\theta)\right]^{T}, \mu_{i}(\theta) \geq 0, \\
\forall i, \sum_{i=1}^{M} \mu_{i}(\theta(t))=1
\end{array}\right\}
$$

\section{POLYTOPIC UNKNOWN INPUT OBSERVER DESIGN}

\subsection{Preliminary study}

In this section, we consider a nonlinear descriptor system represented by the following polytopic LPV descriptor model:

$$
\left\{\begin{array}{l}
E \dot{x}(t)=\sum_{i=1}^{M} \mu_{i}(\theta(t))\left(A_{i} x(t)+B_{i} u(t)+R_{i} d(t)\right) \\
y(t)=C x(t)
\end{array}\right.
$$

A local model will be defined by:

$$
\left\{\begin{array}{l}
E \dot{x}(t)=A_{i} x(t)+B_{i} u(t)+R_{i} d(t) \\
y(t)=C x(t)
\end{array}\right.
$$

At this step, we will focus on the design of a local observer for this linear descriptor model (6). Since $\operatorname{rank}(E)=r$ and $\operatorname{rank}\left(\left[\begin{array}{ll}E R_{i}\end{array}\right]\right)=\operatorname{rank}(E)$, there exists a non singular matrix $\mathrm{P}$ such as (Darouach et al 1995)

$$
\mathrm{PE}=\left[\begin{array}{c}
E_{0} \\
0
\end{array}\right], \mathrm{PA}_{\mathrm{i}}=\left[\begin{array}{l}
A_{0 i} \\
A_{1 i}
\end{array}\right], \mathrm{PB}_{\mathrm{i}}=\left[\begin{array}{l}
B_{0 i} \\
B_{1 i}
\end{array}\right], \mathrm{PR}_{\mathrm{i}}=\left[\begin{array}{l}
R_{0 i} \\
0
\end{array}\right]
$$

where $E_{0} \in R^{r \times n}$ and $\operatorname{rank}\left(E_{0}\right)=r$. This transformation makes it possible to split the static part and the dynamic part of the descriptor system (6). By injecting the static part in the outputs equations, the submodel (6) becomes

$$
\left\{\begin{array}{l}
E_{0} \dot{x}(t)=A_{0 i} x(t)+B_{0 i} u(t)+R_{0 i} d(t) \\
y_{0 i}(t)=C_{0 i} x(t)
\end{array}\right.
$$

where

$\mathrm{y}_{0 \mathrm{i}}(\mathrm{t})=\left[\begin{array}{l}-B_{1 i} u(t) \\ y(t)\end{array}\right] \in R^{\mathrm{j}}, \mathrm{C}_{0 \mathrm{i}}=\left[\begin{array}{l}A_{1 i} \\ C\end{array}\right] \in R^{\mathrm{jxn}}$ and $j=p+n-r$

The polytopic LPV descriptor model is written as follows

$$
\left\{\begin{array}{l}
E_{0} \dot{x}(t)=\sum_{i=1}^{M} \mu_{i}(\theta(t))\left(A_{0 i} x(t)+B_{0 i} u(t)+R_{0 i} d(t)\right) \\
y_{0}(t)=C_{0} x(t)
\end{array}\right.
$$

where: $\mathrm{y}_{0}(\mathrm{t})=\left[\begin{array}{c}-\sum_{i=1}^{M} \mu_{i}(\theta(t)) B_{1 i} u(t) \\ y(t)\end{array}\right] \in R^{\mathrm{j}}$

and $\mathrm{C}_{0}=\left[\begin{array}{c}\sum_{i=1}^{M} \mu_{i}(\theta(t)) A_{1 i} \\ C\end{array}\right] \in R^{\mathrm{jxn}}$.

\subsection{Polytopic UIO Design}

The proposed polytopic UIO of the polytopic LPV descriptor model (8) has the following form:

$$
\left\{\begin{array}{l}
\dot{Z}(t)=\sum_{i=1}^{M} \mu_{i}(\theta(t))\left(N_{i} Z(t)+G_{1 i} u(t)+L_{i} y_{0 i}(t)\right) \\
\hat{x}(t)=\sum_{i=1}^{M} \mu_{i}(\theta(t))\left(Z(t)+H_{2 i} y_{0 i}(t)\right)
\end{array}\right.
$$

where $\mathrm{Z}(\mathrm{t})$ represents the estimated vector, $G_{1 i} \in R^{n \times m}$ are the gains of the local observers. The equations (10) define a polytopic UIO for descriptor LPV system (4), if the estimation error tends towards zero asymptotically in spite of the presence of unknown inputs on the system.

This polytopic UIO aggregates all the local observer defined at each vertex. The problem consists in synthesizing the parameters $N_{i}, G_{1 i}, L_{i}$ and $H_{2 i}$ such that the polytopic 
UIO satisfies the properties of stability and rate of convergence of the observation error. To estimate the states of system (4), we assume that (Hou et al 1999)

$$
\begin{aligned}
& \operatorname{rank}\left[\begin{array}{ccc}
A_{0 i}^{T} & E_{0}^{T} & C_{0 i}^{T} \\
E_{0}^{T} & 0 & 0
\end{array}\right]=n+\operatorname{rank}\left(E_{0}\right) \\
& \operatorname{rank}\left[\begin{array}{c}
s E_{0}-A_{0 i} \\
C_{0 i}
\end{array}\right]=n, \forall i=1, \ldots, M
\end{aligned}
$$

The matrices of the polytopic UIO can be determined in such a way to enable the asymptotical convergence to zero of the state estimation error for the $i^{t h}$ model, defined by $e_{i}(t)=x(t)-\hat{x}(t)$

$$
e_{i}(t)=\left(I_{n}-H_{2 i} C_{0 i}\right) x(t)-Z(t), \forall i=1, \ldots, M
$$

Let us consider $H_{1 i} \in R^{n \times r}$, such as

$$
H_{1 i} E_{0}=I_{n}-H_{2 i} C_{0 i}
$$

Then, since $\left[\begin{array}{c}E_{0} \\ C_{0 i}\end{array}\right]$ is of full column rank, and using (14), the equality (13) becomes

$$
e_{i}(t)=x(t)-\hat{x}(t)=H_{1 i} E_{0} x(t)-Z(t)
$$

By taking into account the expressions (9), (10) and (15), the dynamics of the estimation error is given by the following equation

$$
\begin{gathered}
\dot{e}(t)=\sum_{i=1}^{M} \mu_{i}(\theta(t))\left\{H_{1 i} E_{0} \dot{x}(t)-\dot{Z}(t)\right\} \\
\dot{e}(t)=\sum_{i=1}^{M} \mu_{i}(\theta(t))\left\{H_{1 i} A_{0 i} x(t)+H_{1 i} B_{0 i} u(t)+H_{1 i} R_{0 i} d(t)\right. \\
\left.-N_{i}\left(H_{1 i} E_{0} x(t)-e(t)\right)-G_{1 i} u(t)-L_{i} y_{0 i}(t)\right\} \\
\dot{e}(t)=\sum_{i=1}^{M} \mu_{i}(\theta(t))\left\{N_{i} e(t)+\left(H_{1 i} A_{0 i}-N_{i} H_{1 i} E_{0}-L_{i} C_{0 i}\right) x(t)\right. \\
\left.+\left(H_{1 i} B_{0 i}-G_{1 i}\right) u(t)+H_{1 i} R_{0 i} d(t)\right\}
\end{gathered}
$$

If the following conditions are satisfied:

$$
\begin{gathered}
H_{1} A_{0 i}-N_{i} H_{1 i} E_{0}-L_{i} C_{0 i}=0 \\
G_{1 i}=H_{1 i} B_{0 i} \\
H_{1 i} R_{0 i}=0 \\
H_{1 i} E_{0}=I_{n}-H_{2 i} C_{0 i}
\end{gathered}
$$

Equation (17) is reduced to:

$$
\dot{e}(t)=\sum_{i=1}^{M} \mu_{i}(\theta(t)) N_{i} e(t)
$$

The error of state tends asymptotically towards zero if the matrix $\sum_{i=1}^{M} \mu_{i}(\theta(t)) N_{i}$ is stable.

\section{STABILITY AND CONVERGENCE CONDITION}

The sufficient condition for ensuring the stability of (10) is given by the following theorem.

Theorem 1: The polytopic UIO (10) is asymptotically stable if there exists a common matrix $\mathrm{X}=\mathrm{X}^{\mathrm{T}}>0$ such that

$$
N_{i}^{T} X+X N_{i}<0, \forall i \in\{1, . ., M\}
$$

Proof:

Consider a candidate of quadratic function $V(e(t))=$
$e^{T}(t) X e(t)>0$

Using the equation (22)

$$
\left.\dot{V}(e(t))=\sum_{i=1}^{M} \mu_{i}(\theta(t))\left\{e^{T}(t)\left(N_{i}^{T} X+X N_{i}\right)\right) e(t)\right\}<0
$$

for all $\theta, \sum_{i=1}^{M} \mu_{i}(\theta(t))=1$ and $\mu_{i}(\theta(t)) \geq 0$.

There, for all $e(t) \neq 0,(24)$ is satisfied if

$N_{i}^{T} X+X N_{i}<0, \forall i \in\{1, \ldots, M\}$

Definition: The state estimation error between the polytopic LPV descriptor system (9) and the polytopic UIO (10) converges towards zero, if all the pairs $\left(\mathrm{A}_{0 \mathrm{i}}, \mathrm{C}_{0 \mathrm{i}}\right)$ are observable and if the conditions (18) to (21) hold true and

$$
N_{i}^{T} X+X N_{i}<0, \forall i \in\{1, \ldots, M\}
$$

The substitution of (21) in (18) gives

$$
\begin{gathered}
N_{i}=H_{1 i} A_{0 i}+\left(N_{i} H_{2 i}-L_{i}\right) C_{0 i} \\
N_{i}=H_{1 i} A_{0 i}+K_{i} C_{0 i} \text { where } K_{i}=N_{i} H_{2 i}-L_{i}
\end{gathered}
$$

The inequality (25) becomes

$$
\begin{gathered}
\left(H_{1 i} A_{0 i}+K_{i} C_{0 i}\right)^{T} X+X\left(H_{1 i} A_{0 i}+K_{i} C_{0 i}\right)<0, \\
\forall i \in 1, \ldots, M
\end{gathered}
$$

If one considers the change of variable according to $W_{i}=X K_{i}$, (28) becomes:

$$
\begin{gathered}
\left(H_{1 i} A_{0 i}\right)^{T} X+X\left(H_{1 i} A_{0 i}\right)+W_{i} C_{0 i}+C_{0 i}^{T} W_{i}^{T}<0, \\
\forall i \in 1, . ., M
\end{gathered}
$$

These last inequalities are linear compared to the unknown variables $\mathrm{X}$ and $W_{i}$. Consequently, LMI tools can be used to solve (29). The other matrices of the polytopic observer can then be deduced from the matrices $H_{1 i}, H_{2 i}, X$ and $W_{i}$

$$
\begin{gathered}
K_{i}=X^{-1} W_{i} \\
N_{i}=H_{1 i} A_{0 i}+K_{i} C_{0 i} \\
L_{i}=N_{i} H_{2 i}-K_{i} \\
G_{1 i}=H_{1 i} B_{0 i} \\
\text { where }\left[\begin{array}{ll}
H_{1 i} & H_{2 i}
\end{array}\right]=\left[\begin{array}{ll}
I_{n} & 0
\end{array}\right]\left[\begin{array}{cc}
E_{0} & R_{0 i} \\
C_{0 i} & 0
\end{array}\right]^{+}
\end{gathered}
$$

The polytopic unknown inputs observer is obtained by the interpolation of the observers from each vertex by using the same validity functions as the polytopic LPV descriptor system.

Remark: To ensure the rate of estimation error convergence, one defines in the left part of the complex plan a bounded area $\mathrm{S}$ with a line of abscissa $(-\alpha)$ where $\alpha \in R^{+}$. The inequalities (29) must be replaced by these inequalities

$$
\begin{gathered}
\left(H_{1 i} A_{0 i}\right)^{T} X+X\left(H_{1 i} A_{0 i}\right)+W_{i} C_{0 i}+C_{O i}^{T} W_{i}^{T}+2 \alpha X<0 \\
\forall i \in 1, \ldots, M
\end{gathered}
$$

\section{FAULT DETECTION AND ISOLATION FOR LPV DESCRIPTOR SYSTEM}

A method of Fault Detection, Isolation (FDI) and estimation with a polytopic UIO has been given by (Rodrigues et al 2005). In this section, we will extend this approach to affine LPV descriptor system. Assume that the affine LPV descriptor system (8) is affected by an actuator fault vector $f \in R^{f}$. 


\subsection{Residual generation and actuator fault}

Here, a residual generation using unknown input observer scheme is considered in order to be sensitive to fault vector $f(t)$ and insensitive to $f_{d}(t)$ like in a Generalized Observer Scheme (see (Theilliol et al. 2002)) . We consider that only a single actuator fault may occur at a given time, simultaneous faults are not considered. Hence, vector $f_{d}(t)$ is considered in this FDI scheme as an unknown input. In order to be able to detect and isolate an actuator fault, we have to consider that the control matrix $B_{0 i}$ is constant, i.e. $B_{0 i}=B_{0}$. Then, the system become as follows

$$
\left\{\begin{array}{l}
E_{0} \dot{x}(t)=\sum_{i=1}^{M} \mu_{i}(\theta(t))\left(A_{0 i} x(t)+B_{0} u(t)+R_{0} f_{d}(t)+F_{0} f(t)\right) \\
y_{0}(t)=C_{0} x(t)
\end{array}\right.
$$

where $R_{0}$ is an $i^{\text {th }}$ column of $B_{0}$ and $F_{0}$ is the matrix $B_{0}$ without the $i^{\text {th }}$ column.

A full-order UIO observer for residual generation has the following form

$$
\left\{\begin{array}{l}
\dot{Z}(t)=\sum_{i=1}^{M} \mu_{i}(\theta(t))\left(N_{i} Z(t)+G_{1 i} u(t)+L_{i} y_{0}(t)\right) \\
\hat{x}(t)=\sum_{i=1}^{M} \mu_{i}(\theta(t))\left(Z(t)+H_{2 i} y_{0}(t)\right) \\
\hat{y}_{0}(t)=C_{0} \hat{x}(t)
\end{array}\right.
$$

Our aim is to generate a residual $r(t)$ which allows to detect actuator fault. Defining the state estimation and output estimation error as $e(t)=x(t)-\hat{x}(t)$ and $e_{y}(t)=$ $y_{0}(t)-\hat{y}_{0}(t)$ respectively, the estimation error equation is described by

$$
\dot{e}(t)=\sum_{i=1}^{M} \mu_{i}(\theta(t))\left(N_{i} e(t)+H_{1 i} F_{0} f(t)\right)
$$

A general expression for the residual vector is written as

$$
r(t)=e_{y}(t)=C_{0} e(t)
$$

Some relations have to be verified for detection purposes, ensuring that such synthesis does not affect fault detection:

$$
\operatorname{rank}\left(H_{1 i} F_{0}\right)=\operatorname{rank}\left(F_{0}\right)
$$

This condition allows to verify that de-coupling does not affect fault detection by the estimation error (Rodrigues et al 2005). These residuals are used to detect and identify the actuator faults. The fault isolation is realized by a bank of $p$ polytopic unknown input observers. Each residual vector $r(t)^{l}$ with $(l \in[1, \ldots, p])$, produced by the l-th polytopic unknown input observer, may be used to detect a fault according to a statistical test: Page-Hinkley test, limit checking test, generalized likelihood ratio test. If conditions (18-21) are fulfilled, an unknown input observer provides an estimation of the state vector, used to generate a residual vector $r(t)^{l}=y(t)(l \in[1, \ldots, p])$, independent from $f_{d}(t)$. This means that $r(t)=0$ if $f(t)=0$ and $r(t) \neq 0$ if $f(t) \neq 0$ whatever $u(t)$ and $f_{d}(t)$.

\subsection{Fault estimation}

According to the fault isolation, the fault magnitude estimation of the corrupted element is extracted directly from the l-th polytopic unknown input observer which is built to be insensitive to the l-th fault $(f(t)=0)$. To estimate the faults, let us replace $x(t)$ by $\hat{x}(t)$ in (36) when $f(t)=0$, the fault $f_{d}(t)$ can be replaced also by $\hat{f}_{d}(t)$ and we obtain

$$
\left\{\begin{array}{l}
E_{0} \dot{\hat{x}}(t)=\sum_{i=1}^{M} \mu_{i}(\theta(t))\left(A_{0 i} \hat{x}(t)+B_{0} u(t)+R_{0} \hat{f}_{d}(t)\right) \\
y_{0}(t)=C_{0} \hat{x}(t)
\end{array}\right.
$$

The estimation $\hat{f}_{d}(t)$ of the unknown input $f_{d}(t)$ is considered by using the whole of the equations (36)

$$
\hat{f}_{d}(t)=R_{0}^{+}\left\{E_{0} \dot{\hat{x}}(t)-\sum_{i=1}^{M} \mu_{i}(\theta(t))\left(A_{0 i} \hat{x}(t)+B_{0} u(t)\right)\right\}
$$

The existence of $\hat{f}_{d}(t)$ is ensured by condition (11), and $R_{0}$ is of full column rank.

\section{ILLUSTRATIVE EXAMPLE}

Let us consider the descriptor LPV system defined by

$$
\left\{\begin{array}{l}
E \dot{x}(t)=A(\theta) x(t)+B(\theta) u(t)+R(\theta) d(t) \\
y(t)=C x(t)
\end{array}\right.
$$

where

$$
\begin{aligned}
& A(\theta)=\left[\begin{array}{cccc}
-0.75 & 1 & 0 & 0 \\
-1 & -0.85+\theta_{1} & 0 & 0 \\
0 & -1 & -0.75+\theta_{1} & 0 \\
0 & 0 & 0 & -1+\theta_{2}
\end{array}\right], \\
& E=\left[\begin{array}{llll}
1 & 0 & 0 & 0 \\
0 & 1 & 0 & 0 \\
0 & 0 & 0 & 0 \\
0 & 0 & 0 & 0
\end{array}\right], B(\theta)=\left[\begin{array}{cc}
1+\theta_{1} & 1 \\
1 & 0.5+\theta_{2} \\
0 & 0 \\
\theta_{2} & 0
\end{array}\right]
\end{aligned}
$$$$
R(\theta)=\left[\begin{array}{c}
1 \\
0.5+\theta_{2} \\
0 \\
0
\end{array}\right] \text { and } C=\left[\begin{array}{llll}
1 & 0 & 0 & 0 \\
0 & 1 & 0 & 1
\end{array}\right]
$$

The variables $\theta_{i}$ vary according to $\theta_{1} \in[-0.05,0.05]$ and $\theta_{2} \in[-0.1,0.1], r=2$. Due to the fact that the system is affine, this system can be represented in a polytopic form. In this fact the representation can be written as follows

$$
\left\{\begin{array}{l}
E \dot{x}(t)=\sum_{i=1}^{M} \mu_{i}(\theta(t))\left(A_{i} x(t)+B_{i} u(t)+R d(t)\right) \\
y(t)=C x(t)
\end{array}\right.
$$

This polytope has 4 vertices corresponding to the extreme values of the parameters $\theta_{i}$. All the matrices describing the system are given.

$$
\begin{aligned}
& A_{1}=\left[\begin{array}{cccc}
-0.75 & 1 & 0 & 0 \\
-1 & -0.9 & 0 & 0 \\
0 & -1 & -0.8 & 0 \\
0 & 0 & 0 & -1.1
\end{array}\right], A_{2}=\left[\begin{array}{cccc}
-0.75 & 1 & 0 & 0 \\
-1 & -0.9 & 0 & 0 \\
0 & -1 & -0.8 & 0 \\
0 & 0 & 0 & -0.9
\end{array}\right] \\
& A_{3}=\left[\begin{array}{cccc}
-0.75 & 1 & 0 & 0 \\
-1 & -0.8 & 0 & 0 \\
0 & -1 & -0.7 & 0 \\
0 & 0 & 0 & -1.1
\end{array}\right], A_{4}=\left[\begin{array}{cccc}
-0.75 & 1 & 0 & 0 \\
-1 & -0.8 & 0 & 0 \\
0 & -1 & -0.7 & 0 \\
0 & 0 & 0 & -0.9
\end{array}\right] \\
& B_{1}=\left[\begin{array}{cc}
0.95 & 1 \\
1 & 0.4 \\
0 & 0 \\
-0.1 & 0
\end{array}\right], B_{2}=\left[\begin{array}{cc}
0.95 & 1 \\
1 & 0.6 \\
0 & 0 \\
0.1 & 0
\end{array}\right], B_{3}=\left[\begin{array}{cc}
1.05 & 1 \\
1 & 0.4 \\
0 & 0 \\
-0.1 & 0
\end{array}\right] \\
& B_{4}=\left[\begin{array}{cc}
1.05 & 1 \\
1 & 0.6 \\
0 & 0 \\
0.1 & 0
\end{array}\right], R_{1}=R_{3}=\left[\begin{array}{c}
1 \\
0.4 \\
0 \\
0
\end{array}\right], R_{2}=R_{4}=\left[\begin{array}{c}
1 \\
0.6 \\
0 \\
0
\end{array}\right]
\end{aligned}
$$

and $C_{1}=C_{2}=C_{3}=C_{4}=C=\left[\begin{array}{llll}1 & 0 & 0 & 0 \\ 0 & 1 & 0 & 1\end{array}\right]$

The parameters $\mu_{i}(\theta)$ are:

$\mu_{1}(\theta)=\frac{\theta_{1}-\underline{\theta}_{1}}{\bar{\theta}_{1}-\underline{\theta}_{1}} \frac{\theta_{2}-\underline{\theta}_{2}}{\bar{\theta}_{2}-\underline{\theta}_{2}}=\frac{\left(\theta_{1}+0.05\right)\left(\theta_{2}+0.1\right)}{0.02}$ 


$$
\begin{aligned}
& \mu_{2}(\theta)=\frac{\theta_{1}-\underline{\theta}_{1}}{\bar{\theta}_{1}-\underline{\theta}_{1}} \frac{\bar{\theta}_{2}-\theta_{2}}{\bar{\theta}_{2}-\underline{\theta}_{2}}=\frac{\left(\theta_{1}+0.05\right)\left(0.1-\theta_{2}\right)}{0.02} \\
& \mu_{3}(\theta)=\frac{\bar{\theta}_{1}-\theta_{1}}{\bar{\theta}_{1}-\underline{\theta}_{1}} \frac{\theta_{2}-\underline{\theta}_{2}}{\bar{\theta}_{2}-\underline{\theta}_{2}}=\frac{\left(0.05-\theta_{1}\right)\left(\theta_{2}+0.1\right)}{0.02}
\end{aligned}
$$

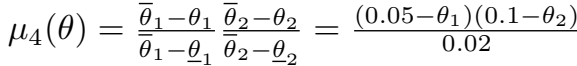

Those models can be transformed as follows $E_{0}=$ $\left[\begin{array}{llll}1 & 0 & 0 & 0 \\ 0 & 1 & 0 & 0\end{array}\right], A_{01}=A_{02}=\left[\begin{array}{cccc}-0.75 & 1 & 0 & 0 \\ -1 & -0.9 & 0 & 0\end{array}\right]$

$$
\begin{aligned}
& A_{03}=A_{04}=\left[\begin{array}{cccc}
-0.75 & 1 & 0 & 0 \\
-1 & -0.8 & 0 & 0
\end{array}\right], B_{01}=\left[\begin{array}{ll}
0.95 & 1 \\
1 & 0.4
\end{array}\right] \\
& B_{02}=\left[\begin{array}{cc}
0.95 & 1 \\
1 & 0.6
\end{array}\right], B_{03}=\left[\begin{array}{ll}
1.05 & 1 \\
1 & 0.4
\end{array}\right], B_{04}=\left[\begin{array}{ll}
1.05 & 1 \\
1 & 0.6
\end{array}\right] \\
& C_{01}=\left[\begin{array}{cccc}
0 & -1 & -0.8 & 0 \\
0 & 0 & 0 & -1.1 \\
1 & 0 & 0 & 0 \\
0 & 1 & 0 & 1
\end{array}\right], C_{02}=\left[\begin{array}{cccc}
0 & -1 & -0.8 & 0 \\
0 & 0 & 0 & -0.9 \\
1 & 0 & 0 & 0 \\
0 & 1 & 0 & 1
\end{array}\right] \\
& C_{03}=\left[\begin{array}{cccc}
0 & -1 & -0.7 & 0 \\
0 & 0 & 0 & -1.1 \\
1 & 0 & 0 & 0 \\
0 & 1 & 0 & 1
\end{array}\right], C_{04}=\left[\begin{array}{cccc}
0 & -1 & -0.7 & 0 \\
0 & 0 & 0 & -0.9 \\
1 & 0 & 0 & 0 \\
0 & 1 & 0 & 1
\end{array}\right] \\
& R_{01}=R_{03}=\left[\begin{array}{l}
1 \\
0.4
\end{array}\right], R_{02}=R_{04}=\left[\begin{array}{l}
1 \\
0.6
\end{array}\right]
\end{aligned}
$$

\subsection{UIO Design}

To design the polytopic UIO, the constraints (11) and (12) are verified. Matrices $\mathrm{X}$ and $\mathrm{Wi}$ are calculated by resolving the LMIs (35) using LMIs toolbox with $\alpha=4$. So for

$$
\begin{aligned}
K_{1}= & {\left[\begin{array}{cccc}
0.1029 & 0.1444 & -4.5890 & 0.1063 \\
0 & -3.4049 & 0.2376 & -3.7454 \\
5.6250 & 4.2561 & -0.4256 & 4.6817 \\
0 & 3.7805 & -0.1313 & -0.3415
\end{array}\right] } \\
K_{2}= & {\left[\begin{array}{cccc}
0.0992 & 0.1024 & -4.5834 & 0.0343 \\
0 & -4.0582 & 0.1481 & -3.6524 \\
5.6250 & 5.0728 & -0.3091 & 4.5655 \\
0 & 4.4797 & -0.1138 & -0.4683
\end{array}\right] } \\
K_{3}= & {\left[\begin{array}{cccc}
0.1067 & 0.1478 & -4.5890 & 0.1131 \\
0 & -3.4577 & 0.2474 & -3.8034 \\
6.4286 & 4.9395 & -0.5058 & 5.4335 \\
0 & 3.8044 & -0.1343 & -0.3152
\end{array}\right] } \\
K_{4}= & {\left[\begin{array}{cccc}
0.1019 & 0.1059 & -4.5834 & 0.0413 \\
0 & -4.1210 & 0.1590 & -3.7089 \\
6.4286 & 5.8872 & -0.3727 & 5.2985 \\
0 & 4.5144 & -0.1177 & -0.4371
\end{array}\right] }
\end{aligned}
$$

The parameters of the polytopic observer are obtained from equality (31), (32) and (33).

For initial conditions, $x_{0}=\left[\begin{array}{llll}0 & 0 & 0 & 0\end{array}\right]^{T}, \hat{x}_{0}=\left[\begin{array}{llll}5 & 3 & 4 & 1\end{array}\right]^{T}$, $\alpha=4$ and an unknown input applied at $\mathrm{t}=10 \mathrm{~s}$, we have the following figures.

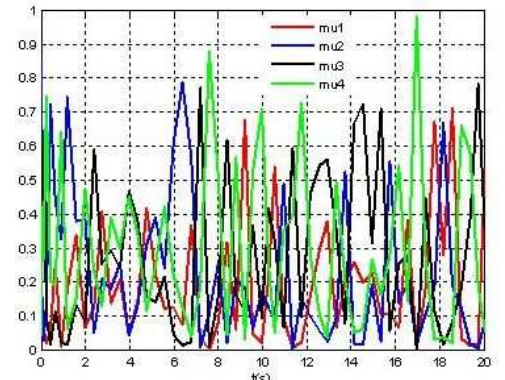

Fig. 1. The parameters evolution $\mu_{i}(t), i \in 1, \ldots, 4$

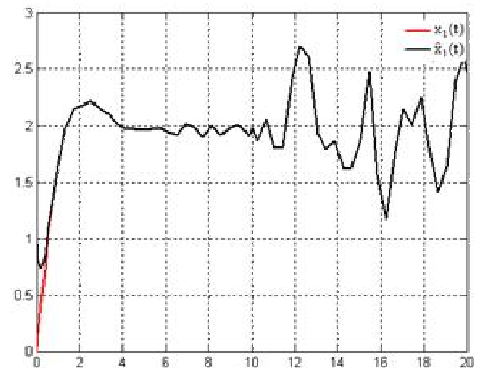

Fig. 2. Asymptotic convergence of the estimated states $\hat{x}_{1}(t)$ towards the real states $x_{1}(t)$

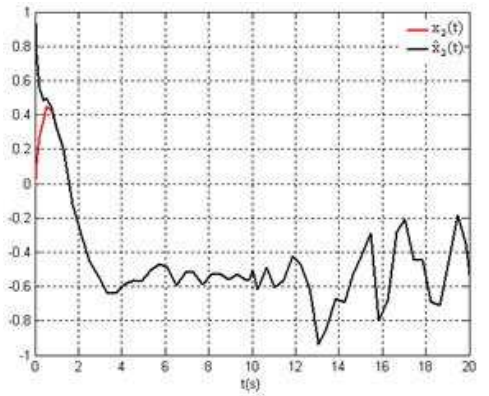

Fig. 3. Asymptotic convergence of the estimated states $\hat{x}_{2}(t)$ towards the real states $x_{2}(t)$

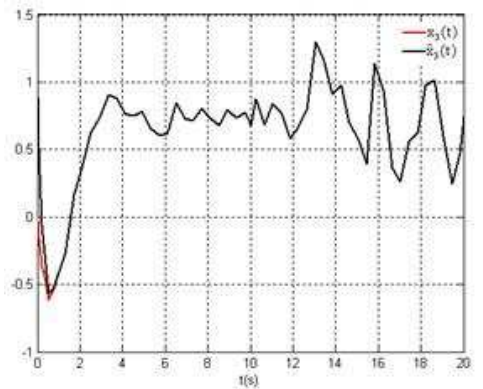

Fig. 4. Asymptotic convergence of the estimated states $\hat{x}_{3}(t)$ towards the real states $x_{3}(t)$

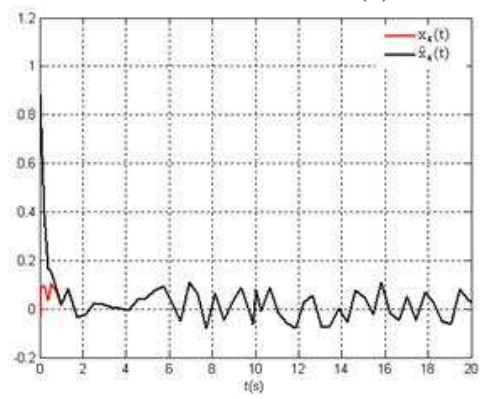

Fig. 5. Asymptotic convergence of the estimated states $\hat{x}_{4}(t)$ towards the real states $x_{4}(t)$

The parameters variations $\mu_{i}(\theta(t))$ are illustrated in Figure (1). The figures (2), (3), (4) and (5) show the comparison between the state of the LPV descriptor system and its estimation from the polytopic UIO. It can be observed that the estimated states can closely track the original states. Next, we will consider a second example for FDI illustration.

\subsection{Fault diagnosis with polytopic LPV UIO}

In this part, we will only consider two actuator faults with constant magnitude. Let us consider the studied plant (36) and the polytopic UIO (37) with the same previous 
matrices except for a constant matrix $B(\theta)$ :

$B_{0 i}=B_{0}=\left[\begin{array}{cc}1 & 1 \\ 1 & 0.5\end{array}\right]$

In this part, we recall that $R_{0}$ is an ith column of $B_{0}$ and $F_{0}$ is the matrix $B_{0}$ without the $i^{\text {th }}$ column (see Section $5)$. In order to detect and isolate the 2 actuator faults, we have to consider 2 polytopic UIO each one designed as explained in Section 5. For the first UIO, we have to consider the following matrices

$R_{0}=\left[\begin{array}{l}1 \\ 1\end{array}\right]$ and $F_{0}=\left[\begin{array}{c}1 \\ 0.5\end{array}\right]$.

For the second UIO, we have to consider the following matrices

$R_{0}=\left[\begin{array}{l}1 \\ 0.5\end{array}\right]$ and $F_{0}=\left[\begin{array}{l}1 \\ 1\end{array}\right]$.

Based on the relation (42), the actuator faults are well estimated on Figures (6) and (7) where we can see the effectiveness of this technique.

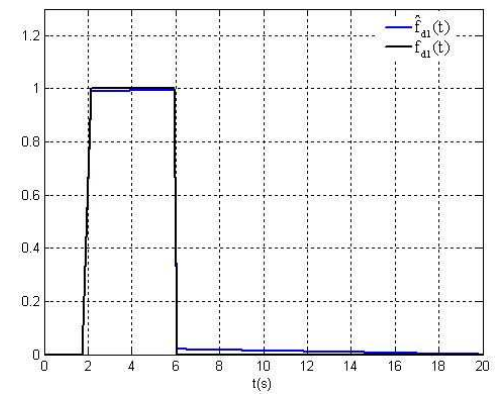

Fig.6. First actuator fault $f_{d 1}(t)$ and its estimation $\hat{f}_{d 1}(t)$

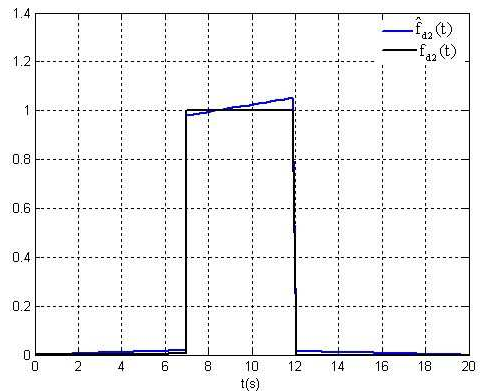

Fig.7. Second actuator fault $f_{d 2}(t)$ and its estimation $\hat{f}_{d 2}(t)$

\section{CONCLUSION}

The main goal of this paper is to design a polytopic Unknown Input Observer for polytopic LPV descriptor systems. This polytopic UIO allows both estimate states in spite of unknown inputs but, it also can be used to detect, isolate and estimate actuator fault. This paper generalizes previous works on UIO to polytopic LPV descriptor systems. The stability of the polytopic UIO is based on the resolution of a LMI problem under structural constraints. An example of a polytopic LPV descriptor system has been presented to illustrate the effectiveness of the scheme.

\section{REFERENCES}

A. Akhenak, M. Chadli, D. Maquin and J. Ragot. State estimation of uncertain multiple model with unknown inputs. 43rd IEEE Conference on Decision and Control December 14-17, pp 35633568,2004

B. Marx, D. Koenig and J. Ragot. Design of observers for TakagiSugeno descriptor systems with unknown inputs and application to fault diagnosis. IET Control Theory Appl., Vol. 1, No. 5, pp. 1487-1495, September 2007

M. Boutayeb and M. Darouach . Observers design for non linear descriptor systems. Proceedings of the 34 th Conference on Decision and Control New Orleans,, pp 2369-2374, LA - December 1995

J. Chen and R.J. Patton Robust model-based fault diagnosis for dynamic systems. Kluwer Academic Publishers 1999.

M. Darouach, M. Boutayeb. Design of Observers for Descriptor Systems. IEEE transactions on Automatic Control. vol. 40. no. 7, pp 1323-1327, July 1995.

Z. Gao and S. X. Ding. Actuator fault robust estimation and fault-tolerant control for a class of nonlinear descriptor systems. Automatica vol. 43, pp 912-920, 2007.

S. Grenaille, D. Henry and A. Zolghadri. A method for designing FDI filters for polytopic LPV models. Safeprocess'06 Beijing, China, 2006

M. Hou, P. C. Muller. Observer Design for Descriptor Systems. IEEE Transactions on Automatic Control,vol. 44, no. 1, pp 164-168, January 1999.

D. Koenig. Observer Design for Unknown Input Nonlinear Descriptor Systems via Convex Optimization. IEEE Transactions on Automatic control, vol. 51, no. 6, pp 1047- 1052, June 2006

M. Rodrigues, D. Theilliol, M. Adam-Medina and D. Sauter. A Fault Detection and Isolation Scheme for Industrial Systems based on Multiple Operating Models. Control Engineering Practice ,Vol 16, p 225-239, 2008

M. Rodrigues, D. Theilliol and D. Sauter. Design of a Robust Polytopic Unknown Input Observer for FDI: Application to Nonlinear Systems described by a Multi-Models Representation. 44th IEEE Conference on Decision and Control and European Control Conference, Sevilla, Spain, pp 6268-6273, December 2005.

M. Rodrigues, D. Theilliol, S. Aberkane and D. Sauter. Fault Tolerant Control Design for Polytopic LPV Systems. Int. Journal. Applied Math. Comput. Sciences, Vol. 17, No. 1, pp 27-37, 2007.

R. Toscano. Robust synthesis of a PID controller by uncertain multimodel approach. Information Sciences Elsevier 2006 .

T. Taniguchi, K. Tanaka, and H. O. Wang. Fuzzy Descriptor Systems and Nonlinear Model Following Control. IEEE Transactions on Fuzzy Systems vol. 8, no. 4, pp 442-452, August 2000.

D. Theilliol and H. Noura and J.C. Ponsart. Fault diagnosis and accommodation of three-tank system based on analytical redundancy. ISA Transactions vol. 41, pp 365-382, 2002.

Y. Wang, Z-Q. Sun and F-C. Sun. Robust Fuzzy Control of a Class of Nonlinear Descriptor Systems with Time-Varying Delay. International Journal of Control, Automation and Systems Vol. 2, No. 1, pp 76-82, March 2004.

A. Zolghadri, D. Henry, S. Grenaille. Fault diagnosis for LPV systems. 16th Mediterranean Conference on Control and Automation, MED'08, Ajaccio, France, June 25-27, 2008. 